\title{
Autophagic disposal of the aggregation-prone protein that causes liver inflammation and carcinogenesis in $\alpha$-1-antitrypsin deficiency
}

\author{
DH Perlmutter
}

$\alpha$-1-Antitrypsin (AT) deficiency is a relatively common autosomal co-dominant disorder, which causes chronic lung and liver disease. A point mutation renders aggregation-prone properties on a hepatic secretory protein in such a way that the mutant protein is retained in the endoplasmic reticulum of hepatocytes rather than secreted into the blood and body fluids where it ordinarily functions as an inhibitor of neutrophil proteases. A loss-of-function mechanism allows neutrophil proteases to degrade the connective tissue matrix of the lung causing chronic emphysema. Accumulation of aggregated mutant AT in the endoplasmic reticulum of hepatocytes causes liver inflammation and carcinogenesis by a gain-of-toxic function mechanism. However, genetic epidemiology studies indicate that many, if not the majority of, affected homozygotes are protected from liver disease by unlinked genetic and/or environmental modifiers. Studies performed over the last several years have demonstrated the importance of autophagy in disposal of mutant, aggregated AT and raise the possibility that predisposition to, or protection from, liver injury and carcinogenesis is determined by the balance of de novo biogenesis of the mutant AT molecule and its autophagic disposal.

Cell Death and Differentiation (2009) 16, 39-45; doi:10.1038/cdd.2008.103; published online 11 July 2008

$\alpha-1$-Antitrypsin (AT) is the archetype of the serpin protein family, most of which are serine protease inhibitors. ${ }^{1}$ AT is the principal blood-borne inhibitor of neutrophil elastase and also inhibits cathepsin $G$ and proteinase 3 , neutrophil-derived proteases that can degrade the major constituents of the lung connective tissue matrix. ${ }^{2}$ It is predominantly synthesized by the liver and, as an acute phase reactant, its plasma concentrations increase significantly during the host response to inflammation and tissue injury. ${ }^{3}$

In the classical form of AT deficiency, which affects 1 in 1800-2000 live births, a single nucleotide substitution results in abnormal folding early in the secretory pathway (reviewed in Perlmutter $\mathrm{DH}^{4}$ ). The mutant protein, called ATZ, has an increased tendency to polymerize and aggregate, ${ }^{5,6}$ with only $10-15 \%$ of newly synthesized molecules able to traverse the secretory pathway and reach the extracellular fluid. The bulk of newly synthesized ATZ is retained in the endoplasmic reticulum (ER). Large polymers have been observed in the ER of liver cells by rotary electron microscopy ${ }^{5}$ and account for the histological hallmark of the disease, intrahepatocytic globules (Figure 1). These globules are now known to represent rough ER dilated by aggregated mutant ATZ.

This deficiency was first discovered as a cause of pulmonary emphysema. ${ }^{7}$ It is now well established that lack of AT permits uninhibited proteolytic destruction of elastin and other connective tissue constituents of the lung matrix. ${ }^{2}$ Cigarette smoking markedly accelerates the rate of development and severity of emphysema in $\alpha$-1-antitrypsin deficiency, presumably because any residual AT is functionally inactivated by phagocyte-derived active oxygen intermediates. ${ }^{8}$

The association of liver disease with AT deficiency was only fully appreciated later. We now know that AT deficiency is the most common genetic cause of liver disease in children. ${ }^{3,4}$ It is also known to cause chronic liver disease and hepatocellular carcinoma first diagnosed in adults. ${ }^{9}$ In contrast to what happens in the lung, liver disease is thought to be caused by a gain-of-toxic function mechanism in which the accumulation of the mutant AT molecule in the ER of liver cells initiates a series of events that are eventually hepatotoxic. The most compelling evidence for the gain-of-toxic function mechanism comes from the observation that mice transgenic for the mutant human ATZ gene develop hepatic inflammation and carcinomas together with the characteristic intrahepatocytic globules. ${ }^{10,11}$ These mice have normal levels of endogenous anti-proteases and so the liver disease must be attributable to a gain-of-toxic function mechanism.

A cohort of homozygotes for the classical form of AT deficiency were identified by an unbiased nationwide newborn

Department of Pediatrics, Cell Biology and Physiology, University of Pittsburgh School of Medicine, Children's Hospital of Pittsburgh of UPMC, 3705 Fifth Avenue, Pittsburgh, PA 15213-2583, USA

Corresponding author: DH Perlmutter, Department of Pediatrics, Cell Biology and Physiology, University of Pittsburgh School of Medicine, Children's Hospital of Pittsburgh of UPMC, 3705 Fifth Avenue, Pittsburgh, PA 15213-2583, USA. Tel: + 412692 8071; Fax: + 412692 5946;

E-mail: david.perlmutter@chp.edu

Keywords: autophagy; $\alpha-1$-antitrypsin deficiency; liver disease; hepatocellular carcinoma; aggregation-prone proteins; protein misfolding

Abbreviations: AT, $\alpha-1$-antitrypsin deficiency; ER, endoplasmic reticulum

Received 31.3.08; revised 03.6.08; accepted 12.6.08; Edited by G Kroemer; published online 11.7.08 


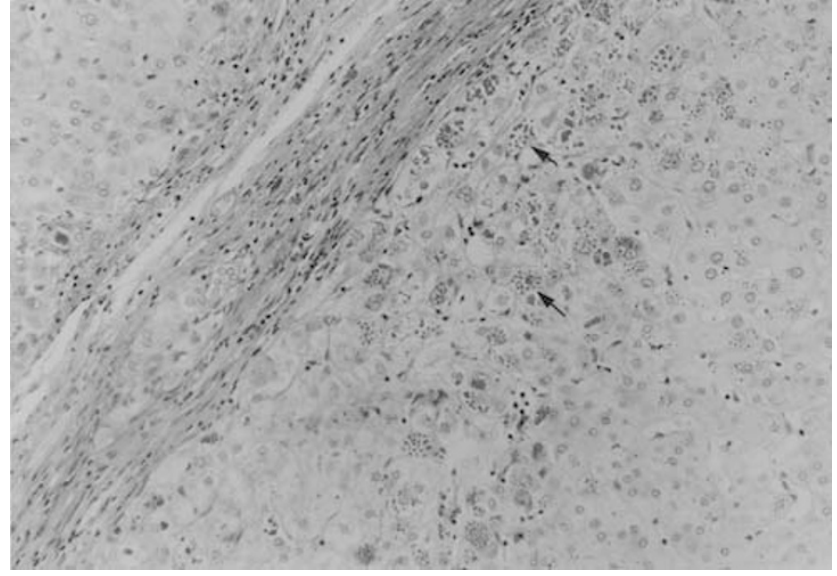

Figure 1 Liver biopsy specimen from a patient with the classical form of AT deficiency. The specimen is stained with periodic acid-Schiff and then treated with diastase so that glycoproteins are identified. Hepatocytes with PAS + globules (arrows) as well as hepatocytes devoid of globules can be seen in this view (see lower right, upper right and upper left quadrants of this view). (Original magnification, $\times 4$ ). Reproduced from Rudnick DA and Perlmutter $\mathrm{DH}^{40}$ with permission

screening study carried out by Sveger in Sweden in the early 1970s. ${ }^{12}$ This cohort has been followed for over 30 years and we now know that only $8-10 \%$ have developed clinically significant liver disease. ${ }^{13}$ These results provide strong evidence for the concept that modifiers, either genetic or environmental, predispose a subgroup of homozygotes to liver disease and/or protect the remainder of the population from liver disease.

Because accumulation of mutant ATZ in the ER causes liver injury by a gain-of-toxic function mechanism, we have theorized that the putative modifiers of the disease affect the pathway, or pathways, responsible for disposal of mutant ATZ. ${ }^{14}$ By this we mean that genetic traits or environmental factors that even subtly alter the function of a putative disposal pathway could increase or decrease the risk of liver disease. This could also include any cellular response pathways that are activated by the accumulation of a mutant aggregation-prone protein in the ER and that might alter the functional activity of a disposal pathway. One study has substantiated this theory by demonstrating a delay in intracellular disposal of ATZ after gene transfer into cell lines from homozygotes with liver disease when compared with cell lines from homozygotes protected from liver disease. ${ }^{15}$ There was a lag in disposal of ATZ in the cell lines from patients with liver disease. These results have focused attention on the pathways for disposal of mutant ATZ after it has accumulated in the ER.

\section{The Proteasomal and Autophagic Pathways Contribute to Disposal of Mutant ATZ}

Studies in yeast as well as in mammalian cell lines demonstrated that the proteasome participates in degradation of mutant ATZ. ${ }^{16-18}$ It is still not clear how the ATZ is transported from the lumen of the ER to the proteasome in the cytoplasm. Although retrograde translocation through the microsomal import channel has been proposed for some ER proteins, there is growing evidence for a mechanism in which

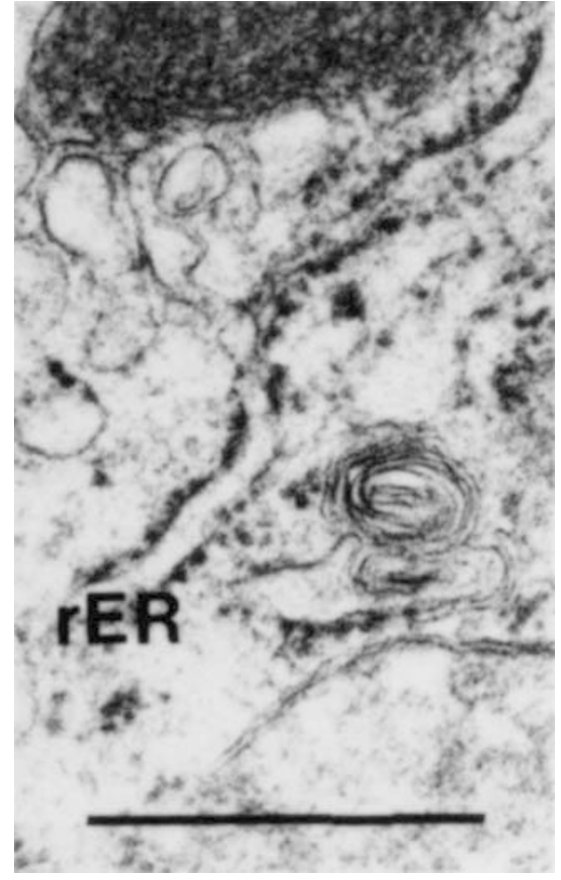

Figure 2 Electron micrograph of a liver biopsy specimen from a patient with the classical form of AT deficiency. An autophagosome pinching off part of the rough endoplasmic reticulum can be visualized. Size bar represents $100 \mathrm{~nm}$. Reproduced from Teckman JH and Perlmutter $\mathrm{DH}^{24}$ with permission

the proteasome, as part of a multiprotein complex that forms a platform on the cytosolic side of the ER membrane, directly mediates extraction of substrates from the ER membrane. ${ }^{19,20}$ Nevertheless, these early studies in yeast, mammalian cell lines and cell-free systems all indicated that the proteasomal pathway could not fully account for disposal of $A T Z^{16-18,21-23}$ - that is in the absence of proteasomal activity there was clear-cut residual and time-dependent degradation of ATZ.

Autophagy was first implicated in AT deficiency when autophagic vesicles were observed in fibroblast cell lines engineered for expression of mutant ATZ. ${ }^{24}$ Several criteria were used to determine that the structures were autophagosomes: ultrastructural characteristics, intravital staining with monodansylcadaverine and immune electron microscopic labeling with DAMP. Furthermore, there was increased accumulation of autophagosomes in the liver cells of the PiZ mouse model of AT deficiency and in the liver cells of patients with AT deficiency ${ }^{24,25}$ (see Figure 2). In these initial studies, the degradation of ATZ in cell line model systems was shown to be partially abrogated by chemical inhibitors of autophagy including 3-methyladenine, LY-294002 and wortmannin. ${ }^{24}$ When taken together with the observation that ATZ was present in autophagosomes by immune electron microscopy, these results suggested that autophagy participated in the disposal of ATZ. However, because the actions of 3-methyladenine, LY-294002 and wortmannin are not specific for autophagy and their influence on degradation of ATZ was partial, a definitive conclusion about the role of autophagy in disposal of ATZ was not reached at that time. 
Ultrastructural studies of the PiZ mouse liver revealed a striking degree of mitochondrial autophagy, or mitophagy. ${ }^{26}$ Mitophagy was also found to be markedly increased in the liver of patients with AT deficiency, even when compared with liver from patients with other hepatic disorders, by quantitative morphometry. Mitochondrial injury was also apparent as evidenced by caspase- 3 activation in the liver in situ and by mitochondrial depolarization in cell lines when ATZ expression is induced. There are at least two possible ways that mitochondrial dysfunction could occur. First, mitochondrial dysfunction could be a direct effect of the 'stressed' ER Indeed, we now know that accumulation of ATZ in the ER activates BAP31, ${ }^{27}$ an integral membrane protein of the ER that mediates pro-apoptotic activity signals to mitochondria. In this case, mitophagy could be activated secondarily to remove damaged mitochondria. A second possible explanation is that mitochondrial dysfunction is an indirect effect of an exuberant autophagic response activated by accumulation of ATZ in the ER - that is mitochondria are innocent bystanders of the effect of ER ATZ accumulation on the autophagic response. To begin to determine which of these two possibilities explain hepatic mitochondrial dysfunction in AT deficiency, we examined the effect of cyclosporine $A$ on cell line and transgenic $\mathrm{PiZ}$ models. ${ }^{26}$ Cyclosporine inhibits the mitochondrial permeability transition but its related compound, tacrolimus, does not. The results showed the cyclosporine, but not tacrolimus, inhibited mitochondrial depolarization, decreased hepatic caspase-3 activation and completely prevented mortality in response to a 72-h fast. There was no change in autophagy. Although definitive testing of this should be done in a system genetically deficient in autophagy, these results are most consistent with the first explanation that mitochondrial injury is a direct result of ER ATZ accumulation and not of an innocent bystander effect of the autophagic response. These results also provided initial evidence for the concept that mitochondrial dysfunction and release of active oxygen intermediates could represent a part of the common final pathway for liver cell injury in AT deficiency. Furthermore, they suggested that cyclosporine A or other drugs that inhibit mitochondrial dysfunction and oxidative mechanisms could be effective therapeutic interventions for liver disease in AT deficiency.

\section{Genetic Evidence for Autophagic Disposal of Mutant ATZ}

To definitively determine whether autophagy contributes to degradation of ATZ it was expressed in an autophagydeficient mammalian cell line, murine embryonic fibroblasts from the atg5-null mouse. ${ }^{28}$ The results demonstrated a significant delay in disposal of ATZ in the atg5-null background compared with the wild-type background. There was progressive accumulation of insoluble ATZ with very large inclusions extending from the ER into the cytoplasm in many of the atg5-null cells. Furthermore, ATZ was immunolocalized to autophagosomes in wild type murine embryonic fibroblasts when autophagosome-lysosome fusion was inhibited by co-expression of dominant-negative Rab 7T22N. Thus, these studies provide definitive genetic evidence that autophagy contributes to degradation of mutant ATZ that accumulates in the ER. Moreover, the results suggest that autophagy plays a homeostatic role in AT deficiency by preventing toxic cytoplasmic accumulation of ATZ through piecemeal digestion of the insoluble aggregates.

To determine whether accumulation of ATZ in the ER activates autophagy we mated our $Z$ mouse with hepatocytespecific inducible expression of ATZ to the GFP-LC3 mouse which renders autophagosomes fluorescent. ${ }^{28}$ Although green fluorescent autophagosomes are seen only in the liver of the GFP-LC3 mouse after starvation, they were observed in the liver of the $Z \times$ GFP-LC3 simply by inducing the expression of the ATZ gene - accumulation of ATZ in the ER was sufficient to activate autophagy; starvation was not required. This effect was found to be specific for the aggregation-prone properties of ATZ because it was not seen when the GFP-LC3 mouse was mated to our Saar mouse, the latter being a mouse with hepatocyte-specific inducible expression of the AT Saar variant that accumulates in the ER but does not polymerize/aggregate. Together, these studies provide powerful and definitive evidence that autophagy is specialized for the pathobiology of AT deficiency in which an aggregation-prone protein accumulates in the ER, activates the autophagic response and autophagy plays a critical role in mediating the disposal of the ATZ aggregates.

A study by Kruse et al. ${ }^{29}$ using a completely different approach and system also demonstrated the critical importance of autophagy in the cellular response to the mutant protein in AT deficiency. In this case, a yeast model system was used. A library of yeast mutants was screened for defects in degradation of human ATZ. One of the mutants corresponded to the yeast homology of autophagy gene atg6. In the absence of atg6 or another autophagy gene atg14 there was a marked delay in degradation of ATZ. The delay in degradation in atg6-deficient or atg14-deficient yeast was particularly apparent at high levels of expression of ATZ as though the proteasomal pathway was sufficient at low levels of expression but autophagy was required at higher levels of expression.

Kruse et al. ${ }^{30}$ also recently discovered that a mutant subunit of fibrinogen that aggregates in the ER of liver cells in an inherited form of fibrinogen deficiency depends on autophagy for disposal. Interestingly, this type of fibrinogen deficiency is associated with a chronic liver disease, providing evidence for the notion that the accumulation of aggregation-prone protein in the ER is hepatotoxic and for the notion that autophagy is particularly specialized as a cellular response to retention of aggregated protein in the ER.

We now envision that when ATZ accumulates in the ER it can be degraded by two major mechanisms, the proteasomal and autophagic pathways. The autophagic pathway is specialized for the polymerized/aggregated forms of ATZ that are accumulating constitutively but become particularly abundant at high levels of expression such as might occur during an acute phase response. The proteasome is probably specialized for the soluble forms of ATZ that accumulate in the ER, presumably bound to multiple chaperones. ${ }^{31}$ Indeed, one could imagine a series of events that occur in the ER: an initial response in which soluble ATZ is bound by the ER chaperones designed to prevent its aggregation; chaperonebound soluble ATZ is directed to the ERAD pathway for 
degradation by the proteasome; when biogenesis and/or accumulation of ATZ exceeds the capacity of chaperones/ ERAD pathway it begins to aggregate, activating autophagy for disposal.

It is important to note that several recent studies on other aggregation-prone proteins have shown that proteasomal function can be impaired by these mutant proteins when they accumulate in the cytoplasm as aggregates. ${ }^{32,33}$ Thus, we could imagine that if autophagy cannot keep up with the formation of aggregated ATZ, such as might occur at high levels of expression, that the aggregated ATZ impairs the proteasome and theoretically lead to a vicious cycle that favors massive intracellular accumulation of ATZ.

The studies of Kruse et al. ${ }^{29}$ have suggested that there is a third pathway for disposal of ATZ in yeast that involves transit to the trans-Golgi and then targeting to the vacuole. A comparable pathway in mammalian cells has not yet been described.

\section{Potential Mechanisms for Activation of Hepatic Autophagy in AT Deficiency}

There is very limited data on the mechanisms by which autophagy is activated in the liver in this disorder. However, recently we discovered by genomic analysis a marked upregulation of regulator of $\mathrm{G}$ signaling 16 (RGS16) and a smaller increase in RGS5 in the liver of the Z mouse, a mouse that has hepatocyte-specific inducible expression of ATZ. ${ }^{34}$ These results were highly statistically significant because they were compared with expression of the same gene products in the liver of the $\mathbf{Z}$ mouse in the uninduced state, in the liver of control mice with hepatocyte-specific inducible expression of the wild-type human AT gene and in the liver of nontransgenic littermates of the same original genetic background. The increase in RGS16 expression was quite marked in magnitude and was apparent at any time during the day, eliminating the possibility that it was because of a peculiar diurnal effect or a difference in feeding associated with the AT deficiency state. The increase in RGS16 expression was also observed in cell line models. It was not observed when cells were treated with drugs that activate the unfolded protein response, including tunicamycin and calcium ionophore A23187. It was not observed in the liver of mouse with hepatocyte-specific inducible expression of the AT Saar variant, which accumulates in the $E R$, activates the unfolded protein response but does not polymerize and does not activate autophagy. RGS16 RNA levels were also significantly elevated in the liver of ATdeficient patients when compared with liver from normal and disease controls. Although we did not originally understand the functional importance of these specific changes in gene expression, a recent study by Gohla et al. ${ }^{35}$ has led to the hypothesis that upregulation of RGS16 plays a role in

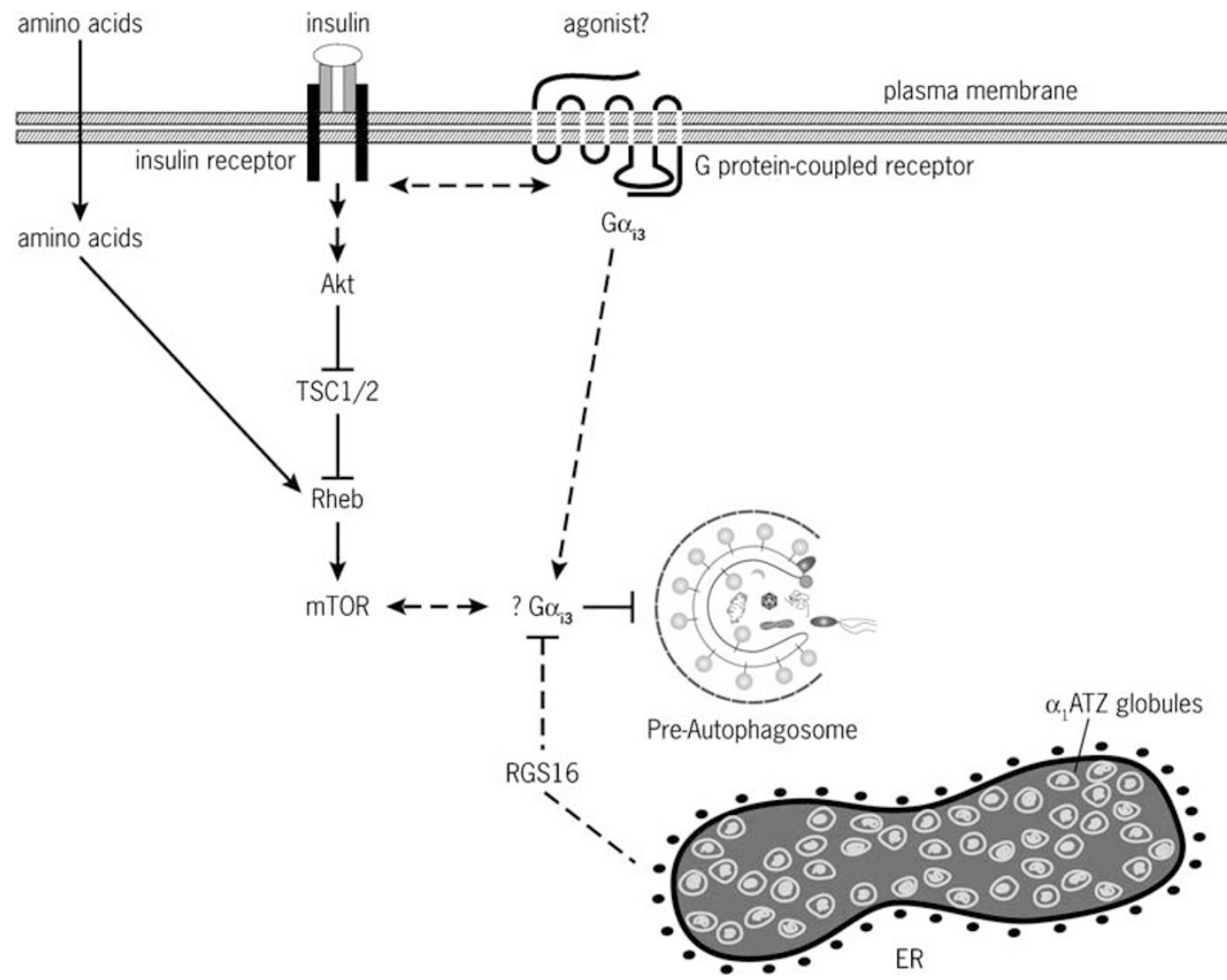

Figure 3 A hypothetical model for the role of RGS16 in activating hepatic autophagy in AT deficiency. The intermediates of the insulin-signaling pathway (Akt, TSC1 and 2, Rheb and mTOR) are shown downstream of the insulin receptor as well as the effect of amino acids. Ordinarily this pathway inhibits the formation of the pre-autophagosome and Goi3 apparently facilitates this inhibitory effect. By antagonizing the activity of Goi3, RGS16 theoretically de-represses the effect of the insulin-signaling pathway on autophagy. In this diagram RGS16 is shown emanating from the ER and Goi3 from the plasma membrane for ease of understanding but the true origin of these molecules as they act on autophagy is not known 
activating the autophagic response. Gohla et al. ${ }^{35}$ showed that targeted disruption of the heterotrimeric $\mathrm{G}$ protein $\mathrm{G} \alpha \mathrm{i} 3$ in a mouse model leads to a marked increase in hepatic insulininduced autophagic activity. This means that Goi3 mediates the hepatic antiautophagic effect of insulin. Because RGS16 (and RGS5) binds to and inhibits the activity of $\mathrm{G} \alpha \mathrm{i} 3,{ }^{36}$ one could infer that upregulation of RGS16 when ATZ accumulates in the liver serves to inhibit signaling downstream from Gai3 and, in so doing, derepress autophagy (Figure 3). Further evidence for this concept comes from studies of another member of the RGS family, AGS3. AGS3 has a GoLoco domain in its carboxyl terminus that shares extensive homology with a domain in RGS16. This domain of AGS3 is responsible for it's binding of Gai3 and for its autophagy-stimulating activity. ${ }^{37}$ Fasting leads to marked induction of hepatic RGS16 gene expression $^{38}$ and is a known stimulus of autophagic activity.

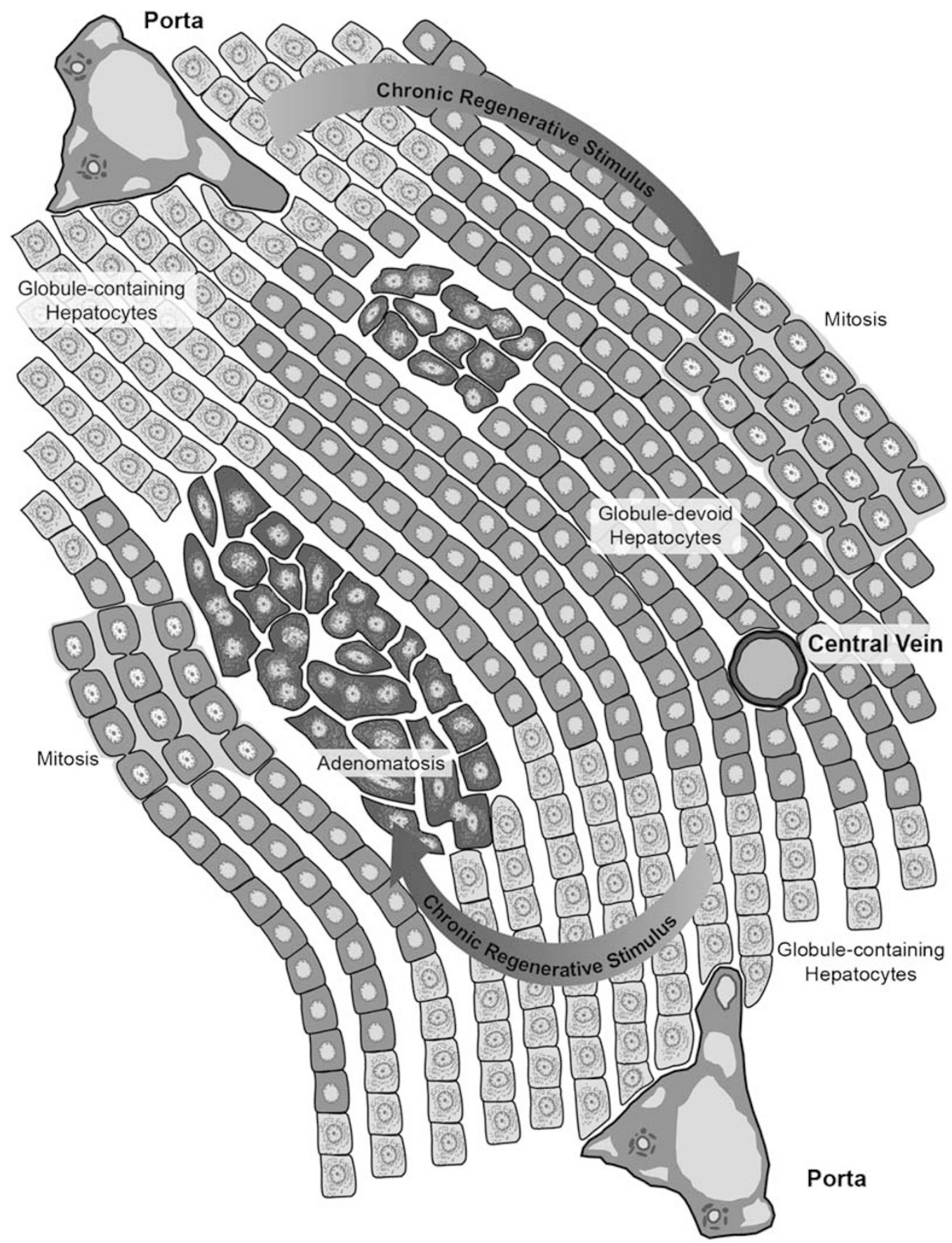

Figure 4 A hypothetical model for carcinogenesis in the liver in AT deficiency. Globule-containing hepatocytes, which tend to be in the periportal region are pale pink because they are 'sick but not yet dead'. They are the source of regenerative signals that only the globule-devoid hepatocytes can respond to. The globule-devoid hepatocytes are deep pink because they are relatively healthy and, with chronic exposure to regenerative signals, can undergo mitosis and ultimately adenomatosis and carcinomatosis. Reproduced from Rudnick DA and Perlmutter $\mathrm{DH}^{40}$ with permission 
Thus, RGS16 is a potential mediator of the autophagic response that is activated by ER accumulation of aggregated ATZ. We doubt that RGS16 is the sole mechanism by which autophagy is activated, particularly as we also see an increase in RGS5 and would predict that RGS5 could provide for compensatory activity if RGS16 were absent. Because activation of autophagy appears to be such an important response when proteins aggregate in the ER one would predict that there are still other mechanisms, including mechanisms outside of the G signaling pathway.

\section{Hepatic Carcinogenesis in AT Deficiency}

There is also relatively little known about the pathogenesis of hepatocellular carcinoma in AT deficiency. One of the possible clues to carcinogenesis comes from the longstanding observation that only some of the hepatocytes have intracellular globules. The presence of globule-containing and globule-devoid hepatocytes has been noted in the liver of patients and in the PiZ transgenic mouse model of AT deficiency. Studies in the PiZ mouse model have shown that there is increased hepatocellular proliferation in the resting liver and that almost all of the proliferating liver cells are globule-devoid. ${ }^{39}$ Consistent with this, almost all of the adenomas and carcinomas that occur in the human disease and in the mouse model arise in the globule-devoid hepatocytic population. ${ }^{40}$ These results have led us to hypothesize (Figure 4) that the pathogenesis of hepatic carcinoma involves the interplay of globule-containing cells that are 'sick but not yet dead' and chronically stimulating the globule-devoid cells 'in trans' in the presence of inflammation. ${ }^{40}$ We know that the globule-devoid cells have accumulated aggregated mutant ATZ ${ }^{41}$ and activated a number of cellular 'alarm' pathways, including ER- and mitochondrial caspases, NF- $\mathrm{BB}$ and autophagy. ${ }^{27}$ Furthermore, we know that the globule-containing cells are relatively impaired in cell proliferation. ${ }^{39}$ Perhaps even more importantly, the regenerative activity in the liver of $\mathrm{PiZ}$ mice is directly proportional to the number of globule-containing cells. ${ }^{39}$ Thus, we believe that these 'sick but not yet dead' globule-containing cells are responsible for regenerative signals but the globule-devoid cells are the ones capable of responding to these signals that is the globule-devoid cells have the selective proliferative advantage. The mechanism by which the globule-devoid cells acquire the selective proliferative advantage is not known but one possibility is that they are younger and have had less time to accumulate ATZ and the damage that accrues from it.

$A$ recent study has provided important new information about cell death in the PiZ mouse liver. ${ }^{42}$ Markers of apoptosis were found to be more pronounced in hepatocytes with greater levels of insoluble ATZ. Furthermore, stimulation of the extrinsic apoptotic pathway with antibody to fas resulted in increased apoptosis almost exclusively within the globulecontaining cells. These results are consistent with the notion that the globule-containing cells are more susceptible to cell death than are the globule-devoid hepatocytes - that is the globule-containing cells are 'sicker' than the globule-devoid hepatocytes. However, these results do not address the possibility that the globule-containing hepatocytes in the AT-deficient liver are relatively resistant to apoptosis when compared with normal hepatocytes. Indeed, the data in this recent study shows an increase in one antiapoptotic protein, cFLIP, in cells with higher levels of insoluble ATZ. ${ }^{42}$ This would mean that the globule-devoid hepatocytes were even more resistant than normal hepatocytes to apoptosis. This is probably an important distinction because relative resistance to apoptosis in either of these cellular compartments could potentially contribute to the mechanism of carcinogenesis.

It is not yet known whether autophagy plays a role in the carcinogenic susceptibility. We know that autophagic activity confers resistance to tumorigenesis. ${ }^{43,44}$ On the basis of our previous work in model cell lines one would predict that there is increased autophagic activity in the globule-containing cells. This prediction should be possible to address by detailed studies in the PiZ $\times$ GFP-LC3 mouse and by mating the PiZ mouse to autophagy-deficient mouse models. It is not clear what happens when the autophagic response is chronically, or even constitutively, activated and whether under those circumstances it might even contribute to the inflammation and carcinogenesis.

Acknowledgements. I am indebted to the US Public Health Service Grants HL037784 and DK052526 for support of research in my lab.

1. Law RH, Zhang Q, McGowan S, Buckle AM, Silverman GA, Wong W et al. An overview of the serpin superfamily. Genome Biol 2006; 7: 216.

2. Crystal RG. Alpha-1-antitrypsin deficiency, emphysema and liver disease: genetic basis and strategies for therapy. J Clin Invest 1990; 95: 1343-1352.

3. Perlmutter $\mathrm{DH}$. Pathogenesis of chronic liver injury and hepatocellular carcinoma in alpha-1-antitrypsin deficiency. Pediatr Res 2006; 60: 233-238.

4. Perlmutter $\mathrm{DH}$. Liver injury in $\alpha 1$-antitrypsin deficiency: an aggregated protein induces mitochondrial injury. J Clin Invest 2002; 110: 579-583.

5. Lomas DA, Evans DL, Finch JT, Carrell RW. The mechanism of $Z \propto 1$-antitrypsin accumulation in the liver. Nature 1992; 357: 605-607.

6. Lomas DA, Mahadeva R. $\alpha 1$-antitrypsin polymerization and the serpinopathies: pathobiology and prospects for therapy. J Clin Invest 2002; 110: 1585-1590.

7. Eriksson S. Pulmonary emphysema and alpha1-antitrypsin deficiency. Acta Med Scand 1964; 175: 197-205

8. Janus ED, Phillips NT, Carrell RW. Smoking, lung function and $\alpha 1$-antitrypsin deficiency. Lancet 1985; 1: 152-154.

9. Eriksson S, Carlson J, Velez R. Risk of cirrhosis and primary liver cancer in $\alpha 1$-antitrypsin deficiency. N Engl J Med 1986; 314: 736-739.

10. Carlson JA, Rogers BB, Sifers RN, Finegold MJ, Clift SM, DeMayo FJ et al. Accumulation of PiZ antitrypsin causes liver damage in transgenic mice. J Clin Invest 1989; 83: 1183-1190.

11. Dycaico MJ, Grant SC, Felts K, Nichols WS, Geller SA, Hager JH et al. Neonatal hepatitis induced by $\alpha 1$-antitrypsin: a transgenic mouse model. Science 1988; 242: 1409-1412.

12. Sveger T. Liver disease in $\alpha 1$-antitrypsin deficiency detected by screening of 200000 infants. N Engl J Med 1976; 294: 1316-1321.

13. Piitulainen E, Carlson J, Ohlsson K, Sveger T. Alpha1-antitrypsin deficiency in 26-year-old subjects: lung, liver, and protease/protease inhibitor studies. Chest 2005; 128: 2076-2081.

14. Teckman JH, Qu D, Perlmutter DH. Molecular pathogenesis of liver disease in alpha1-antitrypsin deficiency. Hepatology 1996; 24: 1504-1516.

15. Wu Y, Whitman I, Molmenti E, Moore K, Hippenmeyer P, Perlmutter D. A lag in intracellular degradation of mutant $\alpha 1$-antitrypsin correlates with the liver disease phenotype in homozygous PiZZ a1-antitrypsin deficiency. Proc Natl Acad Sci USA 1994; 91: 9014-9018.

16. Qu D, Teckman JH, Omura S, Perlmutter DH. Degradation of mutant secretory protein, $\alpha 1$ antitrypsin Z, in the endoplasmic reticulum requires proteasome activity. J Biol Chem 1996; 271: 22791-22295.

17. Werner ED, Brodsky JL, McCracken AA. Proteasome-dependent endoplasmic reticulumassociated protein degradation: an unconventional route to a familiar fate. Proc Natl Acad Sci USA 1996; 93: 13797-13801.

18. Teckman JH, Burrows J, Hidvegi T, Schmidt B, Hale PD, Perlmutter DH. The proteasome participants in degradation of mutant $\alpha 1$-antitrypsin $Z$ in the endoplasmic reticulum of hepatoma-derived hepatocytes. J Biol Chem 2001; 276: 44865-44872.

19. Oberdorf J, Carlson EJ, Skach WR. Uncoupling proteasome peptidase and ATPase activities results in cytosolic release of an ER polytopic protein. J Cell Sci 2006; 119 303-313. 
20. Mayer T, Braun $\mathrm{T}$, Jentsch $\mathrm{S}$. Role of the proteasome in membrane extraction of short-lived ER-transmembrane protein. EMBO J 1998; 17: 3251-3257.

21. Teckman $\mathrm{JH}$, Gilmore R, Perlmutter $\mathrm{DH}$. Role of ubiquitin in proteasomal degradation of mutant $\alpha 1$-antitrypsin $Z$ in the endoplasmic reticulum. Am J Physiol 2000; 278: G39-G48.

22. Cabral CM, Liu Y, Moremen KW, Sifers RN. Organizational diversity among distinc glycoprotein endoplasmic reticulum-associated degradation programs. Mol Biol Cell 2002 13: $2639-2650$

23. Cabral CM, Choudhury $P$, Liu Y, Sifers RN. Processing by endoplasmic reticulum mannosidases partitions a secretion-impaired glycoprotein into distinct disposal pathways. J Biol Chem 2000; 275: 25015-25022.

24. Teckman JH, Perlmutter DH. Retention of mutant $\alpha 1$-antitrypsin $\mathrm{Z}$ in endoplasmic reticulum is associated with an autophagic response. Am J Physiol 2000; 279: G961-G974.

25. Teckman JH, An JK, Loethen S, Perlmutter DH. Fasting in $\alpha 1$-antitrypsin deficient liver constitutive activation of autophagy. Am J Physiol 2002; 283: G1156-G1165.

26. Teckman JH, An JK, Blomenkamp K, Schmidt B, Perlmutter D. Mitochondrial autophagy and injury in the liver in $\alpha 1$-antitrypsin deficiency. Am J Physiol 2004; 286: G851-G862.

27. Hidvegi T, Schmidt BZ, Hale P. Perlmutter DH. Accumulation of mutant alpha-1-antitrypsin $Z$ in the ER activates caspases-4 and -12, NFkB and BAP31 but not the unfolded protein response. J Biol Chem 2005; 280: 39002-39015.

28. Kamimoto T, Shoji S, Mizushima N, Umegayashi K, Hidvegi T, Perlmutter DH et al. Intracellular inclusions containing mutant $\alpha 1$-antitrypsin $Z$ are propagated in the absence of autophagic activity. J Biol Chem 2006; 281: 4467-4476.

29. Kruse KB, Brodsky JL, McCracken AA. Characterization of an ERAD gene as VPS30/ATG6 reveals two alternative and functionally distinct protein quality contro pathways: One for soluble A1PiZ and another for aggregates of A1PiZ. Mol Biol Cell 2006; 17: 203-212.

30. Kruse K, Dear A, Kaltenbrun ER, Crum BE, George PM, Brennan SO et al. Mutan fibrinogen cleared from the endoplasmic reticulum via endoplasmic reticulum-associated protein degradation and autophagy: an explanation for liver disease. Am J Pathol 2006; 168: $1300-1308$.

31. Schmidt BZ, Perlmutter DH. Grp78, Grp94, and Grp170 interact with alpha-1-antitrypsin mutants that are retained in the endoplasmic reticulum. Am J Physiol 2005; 289: G444-G455.

32. Bence NF, Sampat RM, Kopito RR. Impairment of the ubiquitin-proteasome system by protein aggregation. Science 2001; 292: 1552-1555.
33. Layfield R, Cavey JR, Lowe J. Role of ubiquitin-mediated proteolysis in the pathogenesis of neurodegenerative disorders. Ageing Res Rev 2003; 2: 343-356.

34. Hidvegi T, Mirnics K, Hale P, Ewing M, Beckett C, Perlmutter DH. Regulator of G signaling 16 is a marker for the distinct endoplasmic reticulum stress state associated with aggregated mutant $\alpha_{1}$-antitrypsin $\mathrm{Z}$ in the classical form of $\alpha_{1}$-antitrypsin deficiency. J Biol Chem 2007; 282: 27769-27780.

35. Gohla A, Klement K, Piekorz RP, Pexa K, vom Dahl S, Spicher K et al. An obligatory requirement for the heterotrimeric $G$ protein $G_{i 3}$ in the antiautophagic action of insulin in the liver. Proc Natl Acad Sci USA 2007; 104: 3003-3008.

36. Chen C, Sheng-Cai L. The core domain of RGS16 retains G-protein binding and GAP activity in vitro, but is not functional in vivo. FEBS Lett 1998; 422: 359-362.

37. Pattingre S, De Vries L, Bauvy C, Chantret I, Cluzeaud F, Ogier-Denis E et al. The G-protein regulator AGS3 controls an early event during macroautophagy in human intestinal HT-29 cells. J Biol Chem 2003; 278: 20995-21002.

38. Huang J, Pashkov V, Kurrasch DM, Yu K, Gold SJ, Wilkie TM. Feeding and fasting controls liver expression of a regulator of $G$ protein signaling (Rgs16) in periportal hepatocytes. Comp Hepatol 2006; 5: 8-18.

39. Rudnick DA, Liao Y, An JK, Muglia LJ, Perlmutter DH, Teckman JH. Analyses of hepatocellular proliferation in a mouse model of $\alpha 1$-antitrypsin deficiency. Hepatology 2004; 39: 1048-1055.

40. Rudnick DA, Perlmutter DH. Alpha-1-antitrypsin deficiency: a new paradigm for hepatocellular carcinoma in genetic liver disease. Hepatology 2005; 42: 514-521.

41. An JK, Blomenkamp K, Lindblad D, Teckman JH. Quantitative isolation of alpha-1-AT mutant $Z$ protein polymers from human and mouse livers and the effect of heat. Hepatology 2005; 41: 160-167.

42. Lindblad D, Blomenkamp K, Teckman J. Alpha-1-antitrypsin mutant Z protein content in individual hepatocytes correlates with cell death in a mouse model. Hepatology 2007; 46: 1228-1235.

43. Qu X, Yu J, Bhagat G, Furuya N, Hibshoosh H, Troxel A et al. Promotion of tumorigenesis by heterozygous disruption of the beclin1 autophagy gene. J Clin Invest 2003; 112: 1809-1820.

44. Yue Z, Jin S, Yang C, Levine AJ, Heintz N. Beclin 1, an autophagy gene essential for early embryonic development, is a haploinsufficient tumor suppressor. Proc Natl Acad Sci USA 2003; 100: 15077-15082. 